\title{
Meningocele sacro anterior gigante y síndrome de Currarino incompleto en paciente adulta: reporte de un caso
}

Eduardo Jonathan Laos Plasier*1

\section{RESUMEN}

El meningocele sacro anterior es una protrusión del saco dural a través de un defecto óseo de la pared anterior del hueso sacro. Se presenta aislado o asociado a otras malformaciones, como en el síndrome de Currarino, en el que es parte de la triada clásica junto al hemisacro y a una malformación anorrectal, aunque posee gran variabilidad. Presentamos el caso de una mujer adulta con estreñimiento crónico, infección urinaria recurrente y distensión abdominal marcada, que tiene el diagnóstico de meningocele sacro anterior gigante y síndrome de Currarino incompleto. La paciente es sometida a una laminectomía sacra y ligadura del saco tecal a nivel del defecto óseo. Este trabajo describe este inusual tipo de disrafismo espinal oculto, así como la técnica quirúrgica empleada.

Palabras clave: Sacro; Espina bífida oculta; Estreñimiento; Laminectomía (Fuente: DeCS BIREME).

\section{Giant anterior sacral meningocele and incomplete Currarino syndrome in a female adult patient: a case report}

\section{ABSTRACT}

Anterior sacral meningocele is a protrusion of the dural sac through a bone defect in the sacrum anterior wall. It can occur in isolation or be associated with other malformations, such as the Currarino syndrome, which is part of the classic triad together with hemisacrum and anorectal malformation, although it has great variability. This is the case of an adult woman with chronic constipation, recurrent urinary infection and marked abdominal distension, diagnosed with giant anterior sacral meningocele and incomplete Currarino syndrome. The patient underwent a sacral laminectomy and thecal sac ligation at the level of the bone defect. This article describes this rare type of occult spinal dysraphism as well as the surgical technique used.

Keywords: Sacrum; Spina bifida occulta; Constipation; Laminectomy (Source: MeSH NLM).

1 Hospital Nacional Guillermo Almenara Irigoyen, Departamento de Neurocirugía, Servicio de Neurotrauma y Columna, EsSalud. Lima, Perú.

* Autor corresponsal. 


\section{INTRODUCCIÓN}

Los reportes de meningocele sacro anterior (MSA) han sido escasos en el mundo ${ }^{(1,2)}$ (casi 300 casos) ${ }^{(3)}$, y ninguno se ha estudiado en el Perú. El MSA puede aparecer de manera espontánea, pero también como parte de un defecto hereditario de la columna sacrococcígea en el síndrome de Currarino ${ }^{(2,4)}$. Este cuadro fue descrito por primera vez en 1981 por Currarino, quien definió su tríada clásica: defecto óseo sacro, malformación anorrectal (MAR) y masa presacra (MP) (MSA es la más común) ${ }^{(5)}$.

El MSA se caracteriza por una protrusión de la duramadre y de la aracnoides a través de un defecto congénito de la pared anterior del sacro que ocasiona una serie de manifestaciones clínicas por compresión de las estructuras abdominales o pélvicas. No es infrecuente que los MSA permanezcan asintomáticos hasta la adultez (edad media de presentación: 20 a 30 años). Una complicación rara, pero peligrosa, de MSA es la ruptura espontánea del meningocele. Esto conduce a una meningitis séptica, que es particularmente preocupante en las gestantes por la alta probabilidad de ruptura durante el trabajo de parto ${ }^{(4)}$.

El tratamiento del MSA es quirúrgico. Se ha descrito el abordaje anterior, posterior o combinado como tratamiento. El procedimiento usado con más frecuencia es la ligadura del saco en el defecto óseo, a través de laminectomía o laminoplastía sacra, con tasas de mortalidad del 0 al $4 \%$. Se puede considerar aplicar el abordaje anterior, solo o combinado, si el cuello del saco dural que pasa a través del defecto óseo es demasiado ancho para ligarse mediante laminectomía, o si existe una lesión abdominal asociada, por ejemplo, un tumor o una fístula con los órganos genitourinarios o colorrectales ${ }^{(3)}$.

Se presenta el caso de una mujer adulta con los cuadros descritos con la finalidad de considerarlos como diagnósticos diferenciales y, a la vez, describir el abordaje posterior para la ligadura del MSA.

\section{CASO CLÍNICO}

\section{Historia y examen}

Paciente mujer de 42 años de edad, nuligesta y con antecedentes de quiste de ovario desde los 25 años, infección urinaria recurrente y síndrome ansiodepresivo. Se presenta en nuestro hospital con el síntoma principal de distensión abdominal e incomodidad en miembros inferiores sin un territorio radicular definido, cursa con estreñimiento crónico de 15 años de evolución y cefalea a las maniobras de Valsalva. El examen físico no mostró alteración neurológica, pero sí hemiabdomen inferior con gran distensión y cierta resistencia a la palpación. Los exámenes de imágenes mostraron evidencia de MSA, disgenesia sacra, masa quística ovárica izquierda, médula anclada, malformación de Chiari y doble uréter izquierdo (Figuras 1 y 2), sin alteraciones anatómicas digestivas y con la función renal normal, por lo que se diagnóstica MSA gigante tipo I de Cheng y síndrome de Currarino incompleto.
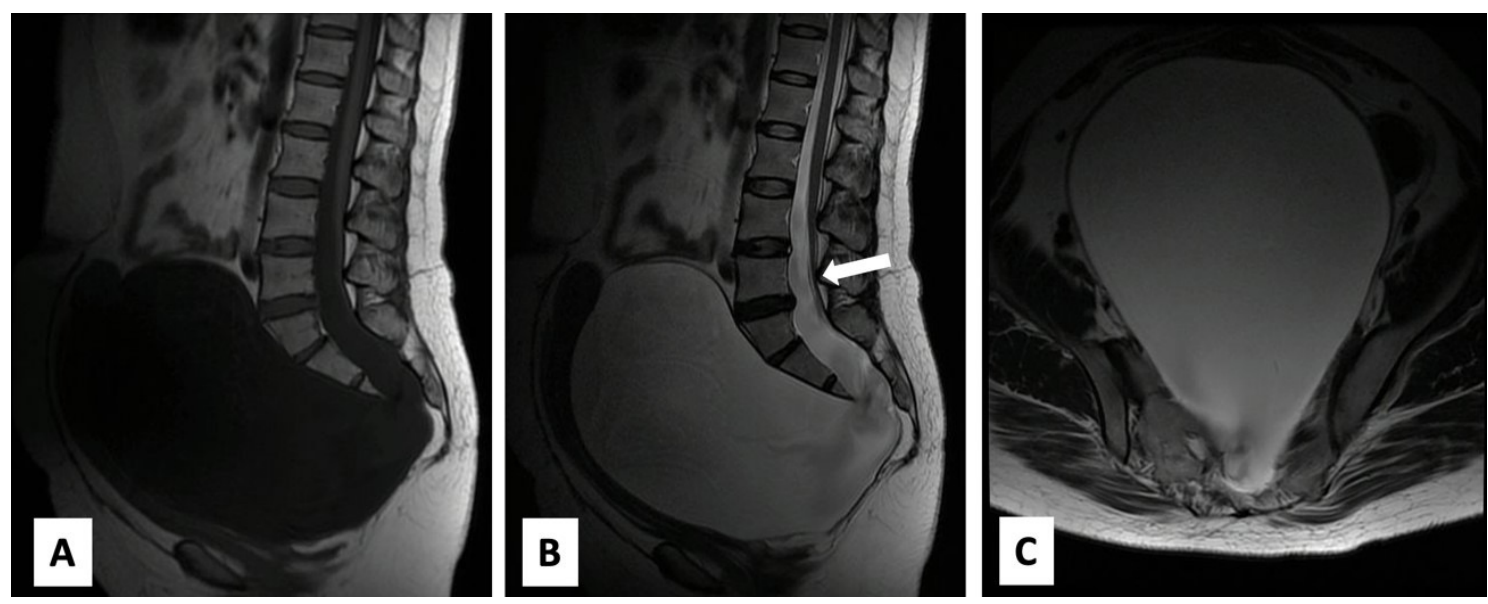

Figura 1. Resonancia magnética (RM) lumbosacra. 1A imagen sagital T1: proceso expansivo hipointenso en cavidad pélvica. 1B Imagen sagital T2: quiste que depende de saco dural a través de un defecto a nivel de S3 de $172 \times 152 \times 129$ mm, también se muestra médula anclada (flecha). 1C Imagen axial T2 a nivel de S3 


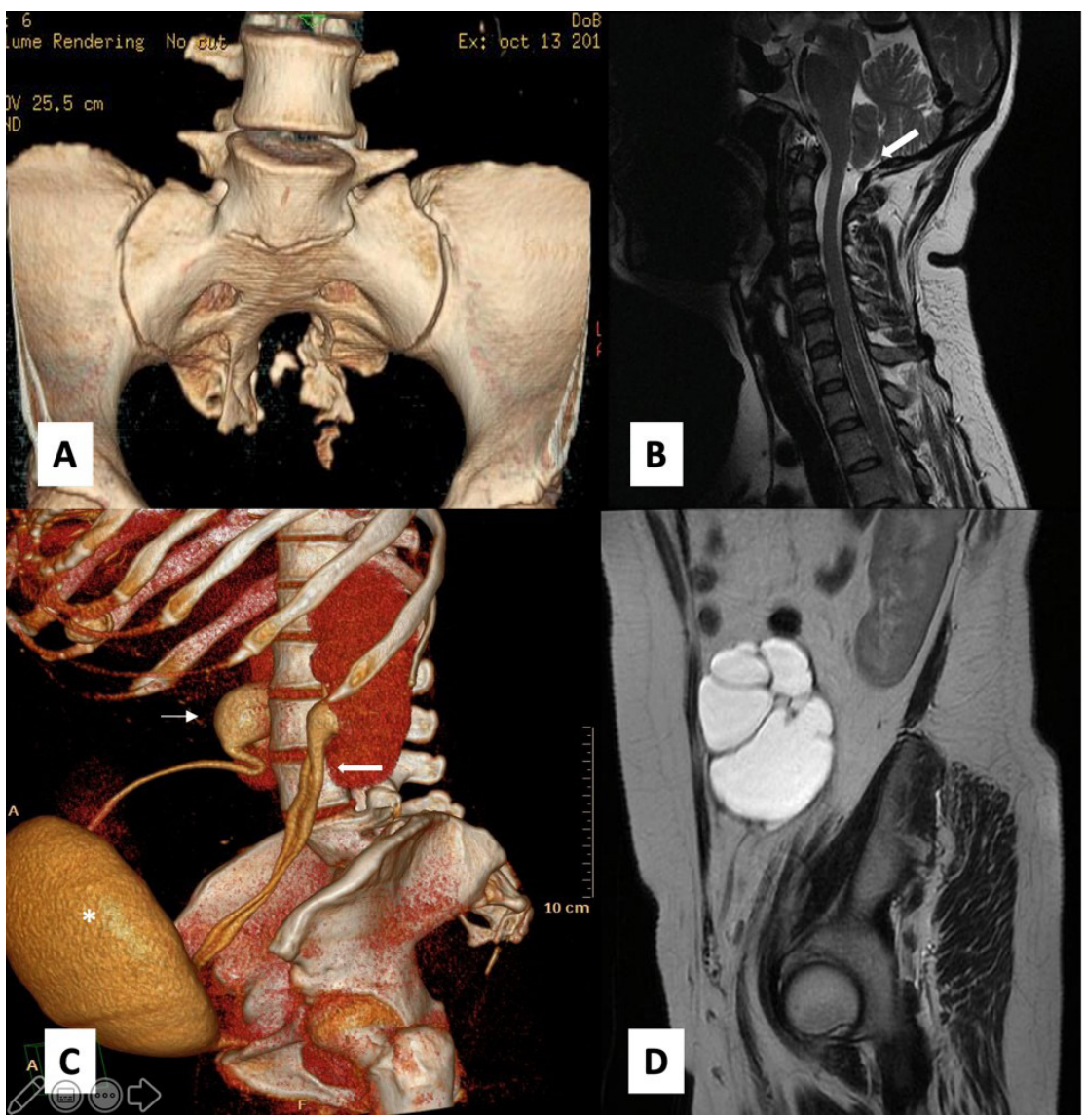

Figura 2. Lesiones asociadas. 2A Tomografía computarizada (TC) reconstrucción 3D que muestra el "sacro en cimitarra". 2B RM en T2: descenso de las amígdalas cerebelosas al límite, $1 \mathrm{~cm}$ por debajo de agujero occipital. 2C Urotomografía: vejiga desplazada y aplanada hacia anterior $\left({ }^{*}\right)$, doble uréter izquierdo (flecha gruesa) e hidronefrosis derecha (flecha delgada). 2D RM en T2: quiste anexial izquierdo

\section{Tratamiento}

El tratamiento que se aplicó fue una laminectomía S1/S2/S3 y luego, con microscopio y monitoreo neurofisiológico de las raíces lumbares bajas y sacras, se seccionó el filum terminale. Después de comprobar que no había raíces dentro del saco herniario con el ostium a la altura de S3, el líquido cefalorraquídeo (LCR) se aspiró de la cavidad. Para terminar, el extremo proximal se liga al saco dural y el defecto óseo queda sellado con fascia muscular, injerto de duramadre y pegamento de tejido (Figura 3).

\section{Evolución}

Luego de la cirugía, la paciente presenta retención urinaria. La estancia hospitalaria fue de 5 días, la herida se conservó en buen estado, la paciente no tuvo fiebre y fue dada de alta. La sonda urinaria se retiró a las 2 semanas. En la evaluación a los tres meses del alta, la paciente informó que el estreñimiento se mantenía, pero no las molestias abdominales, ni la cefalea a maniobras de Valsalva. En la tomografía de control persiste el $30 \%$ del quiste, pero sin variación. En el control de 9 meses aún persiste una imagen de la masa anexial izquierda, la cual es extraída por Ginecología con el diagnóstico patológico de quiste anexial benigno. 


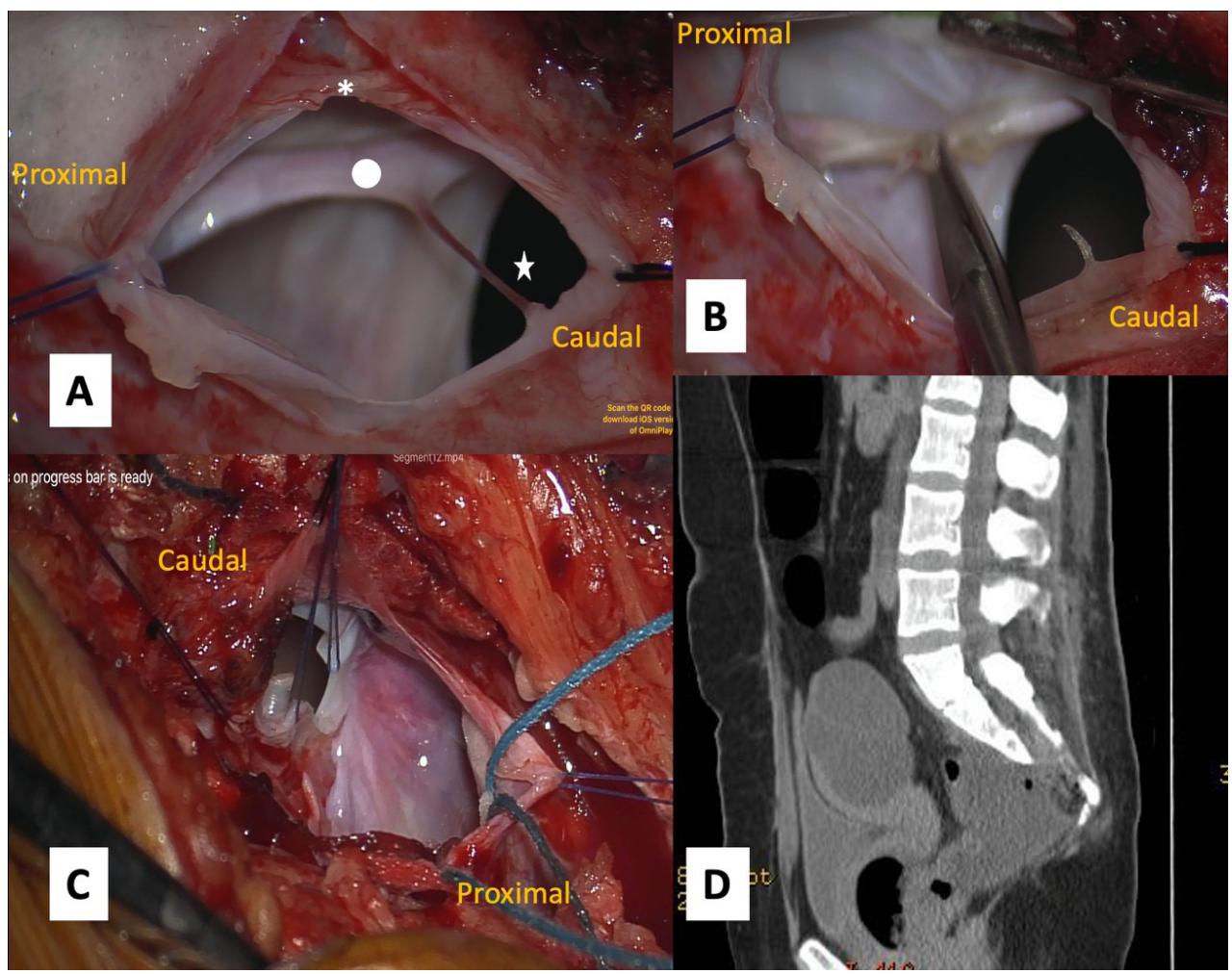

Figura 3. Imágenes intraoperatorias. 3A Previa laminectomía S1, S2 y S3 con la duramadre abierta, se ven raicillas sacras bajas (*), filum terminale (punto blanco) y el defecto por donde se hernia el saco dural hacia ventral a nivel de S3 (estrella). 3B Previa comprobación por monitoreo electrofisiológico y coagulación térmica, se secciona el filum terminale para tratar la médula anclada. 3C Se procede a cerrar segmento proximal y caudal del saco para excluir el saco herniario. 3D Imagen de TC postoperatoria donde se evidencia saco residual del MSA y el quiste anexial izquierdo

\section{DISCUSIÓN}

El presente artículo describe el caso de una mujer adulta con distención abdominal, estreñimiento y cefalea por maniobras de Valsalva. Los exámenes de imagen de la paciente son compatibles con MSA gigante y otras malformaciones del neuroeje y, además, presenta disgenesia sacra y doble uréter izquierdo, lo que configura el síndrome de Currarino incompleto. Como tratamiento se realiza una laminectomía sacra y ligadura del saco tecal a nivel del defecto sacro, con resultados satisfactorios.

\section{Meningocele sacro anterior}

El primer artículo que describe el MSA pertenece a Coller y Jackson ${ }^{(6)}$, y en la literatura existen solo unos 300 casos reportados ${ }^{(3)}$. El MSA se caracteriza por presentar una hernia de la duramadre a través de un defecto congénito en la pared anterior del sacro ${ }^{(2,7,8)}$, resultado probable de una ectasia dural a causa del agrandamiento del espacio subaracnoideo por la pulsación del LCR. Representa, aproximadamente, el $5 \%$ de todas las masas retrorrectales y es más prevalente en las mujeres ${ }^{(9)}$. La mayoría se diagnostican en edad adulta, y es mucho más rara en la infancia ${ }^{(8)}$.

El diagnóstico se realiza con la tomografía computarizada (que proporciona detalles óseos) y la resonancia magnética (RM), que es la prueba de elección (9) (Figura 1). En la radiografía simple, el "sacro en cimitarra", que se observa en el $50 \%$ de los casos, es el hallazgo patognomónico del MSA (7). El diagnóstico diferencial incluye las lesiones quísticas localizadas en la región presacra ${ }^{(9)}$, tumores del tracto gastrointestinal o genitourinario, quistes epidermoides o dermoides, quiste óseo aneurismático, hamartoma, quiste hidatídico, lipoma, linfangioma, quiste perineural, quiste de duplicación rectal, tumores ginecológicos, teratoma o teratocarcinoma. Lo más importante para establecer el diagnóstico definitivo es detectar la comunicación entre la lesión quística y el espacio subaracnoideo, que puede, incluso, requerir de una mielografía. 
El MSA puede aparecer aislado o asociado con el síndrome de Currarino, neurofibromatosis tipo 1, síndrome de Marfan, síndrome Loeys-Dietz, síndrome de Ehlers-Danlos ${ }^{(2,4)}$ y otras anomalías congénitas, como malformaciones anorrectales, malformaciones urogenitales, lipoma, teratoma, tumor epidermoide y quiste dermoide ${ }^{(9)}$. Los adultos pueden ser asintomáticos y el MSA se detecta de manera incidental al realizar una ecografía, una RM lumbosacra o durante una laparotomía exploradora ${ }^{(7)}$. Por lo general, el paciente se vuelve sintomático en la segunda o tercera década de vida ${ }^{(7,9)}$, tal como sucede en nuestro caso, en el cual la primera manifestación fue el estreñimiento, seguido de las infecciones urinarias a repetición y, luego, la distensión abdominal por el crecimiento lento, pero progresivo, del meningocele en la cavidad abdominopélvica. Se describe que la mayoría presenta síntomas compresivos urogenitales y colorrectales (retención urinaria, incontinencia o estreñimiento crónico) o síntomas neurológicos, aunque también pueden debutar como meningitis y problemas obstétricos ${ }^{(2,8)}$.

Cheng et al. (1), en una serie de 11 casos, clasifica al MSA de la siguiente manera: tipo I o caudal, tipo II o paraneural (a través de un foramen sacro) y tipo III o de raíz nerviosa (a través de varios forámenes sacros a la vez). Esta clasificación ayuda a reconocer la relación entre el meningocele y las raíces nerviosas sacras, y a adoptar diferentes estrategias quirúrgicas. Nuestro caso corresponde a un MSA de tipo I, lo cual se comprobó en el intraoperatorio ya que el saco no contenía raíces nerviosas.

\section{Síndrome de Currarino}

El MSA puede ser parte del síndrome de Currarino $(2,5,7,10,11)$. Este cuadro es, en el $70 \%$ de los casos, una enfermedad autosómica dominante con penetrancia variable ligada al gen $H L X B 9$ en la región $7 q 36$, lo que le confiere una amplia variabilidad de genotipo y fenotipo ${ }^{(12,13)}$, y por lo que se puede clasificar en completo e incompleto (11). En 1981, Currarino describe la tríada como un síndrome completo formado por hemisacro o sacro en "cimitarra", malformación anorrectal (MAR) y masa presacra (MP). El tipo de MAR más frecuente es la fístula rectoperineal; sin embargo, diferentes tipos de MAR (fístula rectouretral, fístula rectovestibular, fístula rectoespinal y ano infundibular) pueden presentarse en el síndrome. La MP puede ser MSA, un teratoma, un quiste dermoide, una duplicación rectal u otro tumor no común ${ }^{(10)}$. La MP diferente al MSA obliga a descartar malignidad, ya que se han reportado teratomas malignos, leiomiosarcomas y tumores endocrinos de tipo I (poco frecuentes) (11,12). Pueden aparecer otras anomalías asociadas de diferentes clases como urológicas (doble uréter, doble riñón, reflujo vesicoureteral, riñón displásico o hipospadias), genitales (útero bicorne, vagina septada, genitales ambiguos y clítoris bífido) y del sistema nervioso central (malformación de Arnold Chiari tipo I, médula anclada) ${ }^{(10)}$. Nuestro caso se clasificó como un síndrome de Currarino incompleto ya que presentó solo dos componentes de la tríada: MP (MSA) y hemisacro, y no encontramos una alteración tipo MAR, pero sí presentaba un doble catéter ureteral izquierdo, médula anclada y malformación de Chiari (Figura 2). Cabe señalar que la tríada completa suele diagnosticarse desde el nacimiento, y el síndrome incompleto se detecta en la edad adulta ${ }^{(13)}$.

\section{Complicaciones}

El MSA aumentará de tamaño debido a las pulsaciones del LCR. Esto eleva la presión sobre las estructuras circundantes, lo que conduce a la progresión de los síntomas. Si el cuadro no se trata, puede provocar la ruptura del meningocele y/o fístula rectotecal con una mortalidad del $30 \%$ por meningitis recurrente y sepsis de origen perineal. Además, pueden aparecer complicaciones ginecoobstétricas y urinarias, como infección urinaria recurrente, dispareunia, aborto recurrente o riesgo de parto distócico ${ }^{(3,12)}$. La fístula rectotecal es extremadamente rara (se han reportado solo siete casos) ${ }^{(7)}$. Por último, algunos déficits neurológicos asociados por la médula anclada y la malformación de Chiari han sido descritos ${ }^{(11)}$.

\section{Opciones de tratamiento de MSA}

Las opciones de terapia son variadas y abarcan desde la observación, en los pacientes asintomáticos, hasta un abordaje quirúrgico anterior, posterior o combinado ${ }^{(3)}$. El objetivo del tratamiento quirúrgicoes ocluir la comunicación entre el meningocele y el saco dural para evitar las complicaciones. En los casos sin complicaciones, es seguro realizar un abordaje trans-sacro dorsal con el cierre del pedículo del meningocele. Los principios fundamentales del tratamiento quirúrgico son la delimitación del meningocele, el drenaje de su contenido, la reducción de los elementos neurales (si están involucrados en el saco), la escisión de cualquier tumor asociado y el cierre hermético del defecto dural (1). Rahimizadeh ${ }^{(7)}$ logró una resolución completa del MSA con esta técnica. Recientemente, se describió el cierre laparoscópico transquístico transperitoneal del orificio del meningocele empleando clips de plástico ${ }^{(14)}$. En la serie de Cheng et al. (1), diez de trece pacientes mejoraron sus síntomas a los seis meses del seguimiento, sobre todo, el estreñimiento.

El autor considera que el abordaje trans-sacro dorsal es factible, seguro y con menos complicaciones, por lo cual lo aplicamos en esta paciente. En este caso no se presentaron dificultades, salvo la retención urinaria temporal a causa de la manipulación de raíces sacras bajas.

Este artículo ha descrito un tipo raro de disrafismo oculto asociado a síndrome de Currarino incompleto en una mujer adulta, así como la técnica quirúrgica realizada con éxito.

Contribución de los autores: Eduardo Laos Plasier 
ha participado en concepción y descripción del caso, recolección y obtención de los datos, redacción y revisión crítica del artículo, y en la aprobación de la versión final.

Conflicto de interés: El autor declara no tener conflictos de interés.

Fuente de financiamiento: El artículo ha sido financiado por el autor.

\section{REFERENCIAS BIBILOGRÁFICAS}

1. Cheng C, Tao B, Bai S, Gao G, Li S, Shang A. Anterior sacral meningocele: a new classification and treatment using the dorsal transsacral approach. Spine (Phila Pa 1976). 2020; 45(7): 444-51.

2. Wang $\mathrm{YL}$, He B, Yang J, Xu J, Liu J, Huang S. Individualized management of giant anterior meningoceles-case series. Medicine (Baltimore). 2020; 99(14): e19631.

3. Shastin D, Selvanathan SK, Tyagi AK. A rare case of anterior sacral meningocoele presenting as cauda equina syndrome. $\mathrm{Br} \mathrm{J}$ Neurosurg. 2015; 29(3): 428-9.

4. Paisan GM, Crandall KM, Chen S, Burks SS, Sands LR, Levi AD. Closure of a giant anterior sacral meningocele with an omental flap in a patient with Marfan syndrome: case report. J Neurosurg Spine. 2018; 29(2): 182-6.

5. Aydoseli A, Akcakaya MO, Aras Y, Dolas I, Yanar H, Sencer A. Anterior sacral meningocele in a patient with Currarino Syndrome as a cause of ileus. Br J Neurosurg. 2013; 27(6): 833-5.

6. Shidler FP, Richards V. Anterior sacral meningocele. Ann Surg. 1945; 118(5): 913-8.

7. Rahimizadeh A, Ehteshami S, Rahimizadeh A, Karimi M. Anterior sacral meningocele complicated by rectothecal fistula and rectorrhea: A Case report and review of the literature. Surg Neurol Int. 2020; 11: 117.

8. Mohta A, Das S, Jindal R. Anterior sacral meningocele presenting as constipation. J Pediatr Neurosci. 2011; 6(1): 40-3.

9. Rondina RG, Volpato R, Alves Guerra LF, Nava Martins DL, Bastos Pessanha L. Differential diagnosis of anterior sacral meningocele during the evaluation of post-hysterectomy pelvic collections. Radiol Bras. 2016; 49(3): 203-4.

10. Vélez N, López LS, Rivera JA, Anturi AC, Espinal DA. Currarino's syndrome. A case report. Colomb Médica. 2009; 40(2): 221-5.

11. Tucker AM, Morgenstern P, Diaz D, Sedighim S, Shaul D, Sydorak R, et al. Neurosurgical management of Currarino syndrome: a case series and review of literature. Surg Neurol Int. 2019; 10: 70.

12. Mogollón G, Gutiérrez I. Síndrome de Currarino: Reporte de caso y revisión de diagnóstico, etiología y tratamiento. Ciruped. 2020; 6(1): 26-37.

13. Vinod MS, Chandra Shaw S, Devgan A, Mukherjee S. The Currarino Triad. Med J Armed Forces India. 2018; 74(4): 374-6.

14. Clatterbuck RE, Jackman SV, Kavoussi LR, Long DM. Laparoscopic treatment of an anterior sacral meningocele. Case illustration. J Neurosurg. 2000; 92(Suppl. 2): 246.
Correspondencia:

Eduardo Laos Plasier

Dirección: Av. General Eugenio Garzón 734 Dpto. 608. Jesús

María. Lima, Perú.

Teléfono: 947525439

Correo electrónico: eduplasier@hotmail.com

Recibido: 13 de noviembre de 2020

Evaluado: 25 de enero de 2021

Aprobado: 12 de febrero de 2021

(c) La revista. Publicado por Universidad de San Martín de Porres, Perú. (c) BY Licencia de Creative Commons Artículo en acceso abierto bajo términos de Licencia Creative Commons Atribución 4.0 Internacional. (http://creativecommons.org/licenses/by/4.0/)

ORCID iDs

Eduardo Laos Plasier Thttp://orcid.org/0000-0002-5515-2314 